\title{
Influence of temporal fluctuations and spatial heterogeneity on pollution transport in porous media
}

\author{
Amro M. M. Elfeki • Gerard Uffink • Sophie Lebreton
}

\begin{abstract}
The combined influence of temporal fluctuations and spatial heterogeneity on non-reactive solute transport mechanisms in porous media can be understood by performing simulations of steady and unsteady flow and transport in heterogeneous media. The study focuses on issues such as the degree of heterogeneity, correlation length, separation of the combined effects of temporal and spatial variations, and ergodicity conditions under unsteady flow conditions. It is shown that the effect of temporal variations on solute transport is masked by the strong effect of spatial heterogeneity. There is no obvious difference in plume shape between steady and unsteady flow conditions; the first and the second spatial moments of the plume of the unsteadystate flow condition fluctuate around the steady-state flow condition with the same period of oscillations as the input signal at small storage coefficient $(S \leq 0.001)$. At a relatively high standard deviation in hydraulic conductivity and a small storage coefficient, the unsteady flow condition sharpens the temporal variations in macrodispersion coefficients. The magnitude of the longitudinal macrodispersion coefficient under unsteady flow condition is almost doubled at the maximum values. However, the transverse macrodispersion
\end{abstract}

Received: 17 December 2010 / Accepted: 6 October 2011 Published online: 13 December 2011

(C) Springer-Verlag 2011

Amro M. M. Elfeki is on sabbatical leave from Faculty of Engineering, Mansoura University, Egypt.

\author{
A. M. M. Elfeki $(\varpi)$ \\ Department of Hydrology and Water Resources Management, \\ Faculty of Meteorology, Environment and Arid Land Agriculture, \\ King Abdulaziz University, \\ P.O. Box 80208, Jeddah, Kingdom of Saudi Arabia \\ e-mail: aelfeki@kau.edu.sa \\ Tel.: +966-50-4919254 \\ G. Uffink \\ Geo-Engineering Section, \\ Delft University of Technology, \\ P.O. Box 5048, 2600, Delft, The Netherlands
}

S. Lebreton

Water Resources Section,

Delft University of Technology,

P.O. Box 5048, 2600, Delft, The Netherlands coefficient fluctuates around zero. The Kubo number and Peclet number ranges are 1.2-64 and 10-250, respectively.

Keywords Groundwater flow - Solute transport .

Heterogeneity · Plume spatial moments · Macrodispersion

\section{Introduction}

Flow and transport in natural formations are primarily controlled by the spatial heterogeneity of hydrogeological parameters, especially hydraulic conductivity (Dagan 1984; Tompson and Gelhar 1990; Boggs et al. 1992; Adams and Gelhar 1992, and Gelhar 1993). However, flow and transport processes are not only dependent on spatial heterogeneity but dependent on temporal variability as well. Temporal variability occurs due to seasonal, daily or hourly variations in water levels, piezometric heads or recharge from the land surface (Rehfledt and Gelhar 1992; Dagan et al. 1996; and Wang and Tsay 2001).

Temporal variability that is exhibited in a form of piezometric level fluctuation, acts on the aquifer by modifying the magnitude and direction of groundwater velocities as spatial heterogeneity does; therefore, it has an impact on the transport processes. Temporal variability has been addressed by many researchers, among them Ackerer and Kinzelbach (1985) and Rehfledt and Gelhar (1992). Ackerer and Kinzelbach (1985) showed that temporal fluctuations in the mean hydraulic gradient enhanced transverse mixing. Rehfledt and Gelhar (1992) provide an analysis of the influence of a temporal fluctuation in the direction of the hydraulic gradient on the macrodispersivity tensor in a randomly heterogeneous porous medium. Their analysis indicates that the longitudinal macrodispersivity is negligibly different from that in a steady flow field, while the transverse macrodispersivity is significantly enhanced, which supports the work by Ackerer and Kinzelbach (1985).

Goode and Konikow (1990) have also studied dispersion in transient groundwater flow. They focused on changes in flow direction over time in homogeneous fields, rather than temporal changes in magnitude of velocity, because they claimed that fluctuations in magnitude are unlikely to have a significant effect on dispersion. Yim and Mohsen (1992) have studied the tidal effect on contaminant transport in homogeneous porous media through simulations in onedimension. They concluded that tidal fluctuations cause exit 
concentration levels to be significantly diluted by the surface-water body of the estuary. Wood and Kavvas (1999) have solved the ensemble averaged solute-transport equation for reactive transport in porous media under unsteady flow conditions. They compared their results with previous results from the Borden aquifer in Ontario, Canada (Sudicky 1986). In the Wood and Kavvas (1999) approach, the Darcy scale dispersion is included in the analysis, and it showed that this dispersion term has a small but significant influence on the results. Stochastic analyses of solute dispersion in heterogeneous media under temporal flow fluctuations have been considered by Cirpka and Attinger (2003), and Dentz and Carrera (2005). However, Cirpka and Attinger (2003), and Dentz and Carrera (2005) analyses are based on perturbation methods and focused on the derivation of an up-scaled effective transverse dispersion as the main parameter that is strongly influenced by the temporal fluctuations of the flow field. Uffink et al. (2005) have performed two-dimensional (2D) numerical simulation of steady and unsteady groundwater flow and solute transport as part of the Macrodispersion Experiment, MADE (Boggs et al. 1992). They concluded that the influence of temporal variability is relatively low; spatial variability is the main factor controlling plume spreading.

The current study addresses the combined influence of temporal and spatial variability on contaminant transport in porous media by performing numerical simulations of unsteady flow and transport in heterogeneous porous media. Adapting this approach releases the analysis from any assumptions that might be adopted when using analytical stochastic methods of flow and transport equations. The aim of this work is to study the transport of a non-reactive solute injected into a heterogeneous confined aquifer under temporal variability of the flow field and to investigate the plume behavior in terms of plume shape, plume spatial moments and macrodispersion coefficients for better understanding of the transport mechanisms under these conditions. The study focused on issues that do not get much attention in the literature such as investigating different degrees of heterogeneity in terms of magnitude, separating the combined effects of temporal and spatial variations of the flow fields, estimating macrodispersion coefficients, and considering ergodicity conditions under an unsteady-state flow field.

\section{Setup of flow and transport simulations}

In order to study the effects of spatial aquifer heterogeneity and temporal variability on solute transport by groundwater, a numerical model is set up for that purpose. A heterogeneous aquifer of a constant thickness with impermeable boundaries at the top and the bottom, as shown in Fig. 1, is considered in the analysis. At the left side (upstream boundary), a river with a constant water level is feeding the aquifer. The main direction of the flow of water is from left to right. At the right side

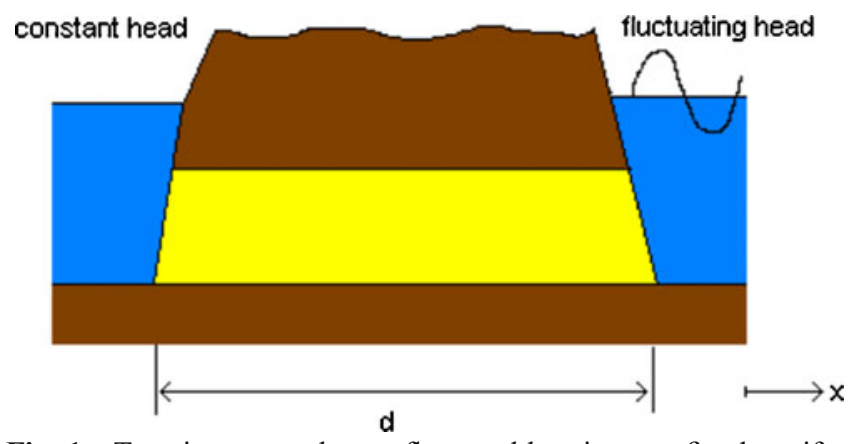

Fig. 1 Transient groundwater flow problem in a confined aquifer: the aquifer cross-section (yellowrefers to the aquifer, reddish-brown is the confining units, bluecorresponds to surface water)

(downstream boundary), the water level is fluctuating with a given function. The influences of these fluctuations on the aquifer response and consequently on the transport of solute are investigated.

The properties of the aquifer such as hydraulic conductivity, $K$, and $S$, storage coefficient, are considered constant over its whole depth, and therefore it is possible to model the aquifer in the horizontal plane with depth-averaged properties. Thus, the problem will be approached in two dimensions. It should also be mentioned that lateral sides of the aquifer are also considered impermeable boundaries.

Numerical simulations of the groundwater flow system and solute transport are performed with two specific numerical models: a groundwater flow model called "TRAN_FLOW" and a solute transport model called "TRAN_SOLUTE". The unsteady groundwater flow model "TRAN $\overline{\text { FLOW" has been }}$ developed by Elfeki (2003). This model is designed to study the influence of transient conditions on the aquifer response (hydraulic heads and Darcy's velocities) of homogeneous and heterogeneous confined aquifers. The model is based on a fully implicit finite difference method and computes hydraulic heads, as well as Darcy's velocities, in horizontal and 2D aquifers. For the sake of completeness, a description is given in the following.

The governing equation, in the absence of source and sink terms, of transient 2D (in the horizontal plane) saturated incompressible fluid flow in an anisotropic heterogeneous confined aquifer is given by:

$S \frac{\partial h(x, y, t)}{\partial t}=\frac{\partial}{\partial x}\left(T_{x x}(x, y) \frac{\partial h(x, y, t)}{\partial x}\right)+\frac{\partial}{\partial y}\left(T_{y y}(x, y) \frac{\partial h(x, y, t)}{\partial y}\right) \in \Omega$

where $T_{\mathrm{xx}}(x, y)$ is the transmissivity in $\mathrm{x}$-direction, $T_{\mathrm{yy}}(x, y)$ is the transmissivity in y-direction, $h(x, y, t)$ is the hydraulic head, $S$ is the storage coefficient, and $\Omega$ is the domain of interest. The transmissivity is related to the hydraulic conductivity by:

$$
\begin{aligned}
& T_{x x}(x, y)=K_{x x}(x, y) H \\
& T_{y y}(x, y)=K_{y y}(x, y) H
\end{aligned}
$$


where $H$ is the aquifer thickness, which is assumed constant in this study. A finite difference model has been developed for discretization of Eq. 1. Detailed derivation of the numerical model is presented in Appendix A.

A large number of solvers are available for systems of linear equations and some of the efficient solvers, in the case of heterogeneous systems with a large number of nodes, are the iterative ones. All the iterative solvers start with an initial guess of the field variable and in each iteration a new and better approximation is computed. It has been proven that the method of conjugate gradient (CG) is powerful in addressing highly heterogeneous media. This method was adopted by Elfeki (1996) for steady-state flow problems. The CG method is extended in the current study to handle time dependent flow problems. The formulas and the algorithm for implementation in the case of transient conditions are presented. The algorithm used here is an extension of the one given by Strikwerda (1989). Some modifications are adopted to handle the heterogeneity of the medium and transient conditions. A fully implicit backward difference scheme that is solved by CG is used for the time integration. This technique is fairly simple, completely stable and is free from oscillation problems. The equations to solve are in the form of a linear system $\mathbf{a x}=\mathbf{b}$ where, $\mathbf{a}$ is the positive definite matrix and the vector $\mathbf{b}$ contains both zeros and the values of the solution on the boundary. The solution algorithm is presented in Appendix B. In the presented algorithm, there is no need to construct the matrix a, however, the algorithm moves through the nodes in the domain until a conversion criterion is achieved.

After the solution of the flow equation, one can calculate the potential head distribution at each time step and consequently the gradient field and the Darcy's velocity field on the grid. This is can be done by differentiation as:

$$
q_{x_{i+1 / 2, j}}^{k+1}=-T_{x x}(x, y)\left(\frac{\partial h(x, y, t)}{\partial x}\right) \approx-T_{x x_{i+1 / 2, j}}\left[\frac{h_{i+1, j}^{k+1}-h_{i, j}^{k+1}}{\Delta x}\right]
$$

$q_{y_{i, j+1 / 2}}^{k+1}=-T_{y y}(x, y)\left(\frac{\partial h(x, y, t)}{\partial y}\right) \approx-T_{y y_{i, j+1 / 2}}\left[\frac{h_{i, j+1}^{k+1}-h_{i, j}^{k+1}}{\Delta y}\right]$

where $q_{x_{i+1 / 2, j}}^{k+1}$ and $q_{y_{i, j+1 / 2}}^{k+1}$ are the inter-nodal Darcy's velocity components between nodes $(i, j)$ and $(i+1, j)$, and between nodes $(i, j)$ and $(i, j+1)$ at time $k+1$. The model has been tested under homogeneous cases where analyt- ical solutions exist and produces accurate results under different values of aquifer properties and excitation parameters (Elfeki et al. 2007).

The transport equation (advection-dispersion equation) of an inert tracer in 2D with full tensor description is given by (Wang and Anderson 1982):

$\frac{\partial C}{\partial t}+\mathrm{V}_{x} \frac{\partial C}{\partial x}+\mathrm{V}_{y} \frac{\partial C}{\partial y}-\frac{\partial}{\partial x}\left(D_{x x} \frac{\partial C}{\partial x}+D_{x y} \frac{\partial C}{\partial y}\right)-\frac{\partial}{\partial y}\left(D_{y x} \frac{\partial C}{\partial x}+D_{y y} \frac{\partial C}{\partial y}\right)=0$

Where, $C$ is the concentration of the solute, and $V_{\mathrm{x}}, V_{\mathrm{y}}$ are the components of the average pore water velocities in the $\mathrm{x}$ - and y-directions respectively at time $t$ :

$\mathrm{V}_{x}=q_{x} n^{-1} H^{-1}, \mathrm{~V}_{y}=q_{y} n^{-1} H^{-1}$

where $n$ is the medium porosity, and $D_{\mathrm{xx}}, D_{\mathrm{xy}}, \mathrm{D}_{\mathrm{yx}}$, and $D_{\mathrm{yy}}$ are the components of the dispersion tensor given by (Bear 1972):

$$
\begin{aligned}
& D_{x x}=\alpha_{L} \mathrm{~V}_{x}{ }^{2}|\mathbf{V}|^{-1}+\alpha_{T} \mathrm{~V}_{y}{ }^{2}|\mathbf{V}|^{-1} \\
& D_{y y}=\alpha_{T} \mathrm{~V}_{x}{ }^{2}|\mathbf{V}|^{-1}+\alpha_{L} \mathrm{~V}_{y}{ }^{2}|\mathbf{V}|^{-1} \\
& D_{x y}=D_{y x}=\left(\alpha_{L}-\alpha_{T}\right) \mathrm{V}_{x} \mathrm{~V}_{y}|\mathbf{V}|^{-1}
\end{aligned}
$$

where, $\alpha_{\mathrm{L}}$ and $\alpha_{\mathrm{T}}$ are longitudinal and transverse porescale dispersivities and $|\mathbf{V}|$ is the resultant velocity given by $|\mathbf{V}|=\sqrt{\mathrm{V}_{x}^{2}+\mathrm{V}_{y}^{2}}$

The transport equation is commonly solved by numerical schemes. Numerical schemes can be classified into Eulerian (e.g. finite difference and finite element methods, see e.g. Wang and Anderson 1982), Lagrangian (e.g. random walk particle tracking, see e.g. Kinzelbach 1986) and Eulerian-Lagrangian (e.g. method of characteristics, see also Kinzelbach 1986) methods. Finite difference and finite element methods are both often used and reliable methods, but in the case of advection dominated transport, they suffer from artificial dispersion (Holly and Usseglio-Polatera 1984). These solution methods produce an extra numerical dispersion which is added to the physical dispersion. Lagrangian methods treat the transport of solute mass as a large number of moving particles and do not solve the advection-dispersion equation directly. The random walk method is the method that is applied in the current code "TRAN_SOLUTE" to solve the transport equation, since the method has some advantages compared with other conventional methods (e.g. finite difference and finite element methods): (1) it provides a good approximation of the dispersion process, (2) it has the ability to solve problems having zeros or low dispersivities, (3) it does not suffer from numerical (artificial) diffusion as other methods do, (4) 
it does not produce negative concentrations, (5) the principle of conservation of mass is satisfied, (6) it is easy to implement and efficient in terms of computer costs, and (7) it is robust and powerful in case of heterogeneous media.
Equation 5 is not solved directly; however, the random walk method is used to simulate the advective-dispersive processes by using a number of particles. The following equations give the $x$ and $y$ coordinates of a particle $p$ at any point in time (Kinzelbach 1986):

$X_{p}(t+\Delta t)=X_{p}(t)+\left(\mathrm{V}_{x}+\frac{\partial D_{x x}}{\partial x}+\frac{\partial D_{x y}}{\partial y}\right) \Delta t+\frac{\mathrm{V}_{x}}{|\mathbf{V}|} Z_{1} \sqrt{2 \alpha_{L}|\mathbf{V}| \Delta t}-\frac{\mathrm{V}_{y}}{|\mathbf{V}|} Z_{2} \sqrt{2 \alpha_{T}|\mathbf{V}| \Delta t}$

$Y_{p}(t+\Delta t)=Y_{p}(t)+\left(\mathrm{V}_{y}+\frac{\partial D_{y x}}{\partial x}+\frac{\partial D_{y y}}{\partial y}\right) \Delta t+\frac{\mathrm{V}_{y}}{|\mathbf{V}|} Z_{1} \sqrt{2 \alpha_{L}|\mathbf{V}| \Delta t}+\frac{\mathrm{V}_{x}}{|\mathbf{V}|} Z_{2} \sqrt{2 \alpha_{T}|\mathbf{V}| \Delta t}$

where, $X_{p}(t+\Delta t)$ and $Y_{p}(t+\Delta t)$ are the new $x$ - and $y$ coordinates of the particle $p, X_{p}(t)$ and $Y_{p}(t)$ are the old $x$ - and $y$-coordinates of the particle $p, Z_{1}$ and $Z_{2}$ are statistically independent normal random numbers with zero mean and unit variance, and $\Delta t$ is the time step in calculations. It is assumed in this study, that the time scale of flow and transport is the same and therefore one single time step was used for both.

In the right hand side of Eqs. 8 and 9, the first term is the old position of the particle; the term between brackets consists of two terms: the advective displacement and the so-called Fokker-Plank term (see Uffink 1990). The last two terms are the dispersive components projected in the $\mathrm{x}$ - and $\mathrm{y}$-directions respectively. Note that these two terms are stochastic and depend on the random numbers $Z_{1}$ and $Z_{2}$. The particle tracking model has been tested for several cases under homogeneous and layered system by Elfeki (1996). In order to quantify the plume behavior, some plume characteristics are calculated. Statistical moments of the plumes (Freyberg 1986) are estimated. These moments are shown in Fig. 2.

The centroid displacement is the average position of the particles. The mean $x$-coordinate $\bar{X}$ and the $y$ coordinate $\bar{Y}$ are computed from the particle cloud as follows.
The $x$-coordinate of the plume centroid is: $\bar{X}(t)=$ $\frac{1}{N_{p}} \sum_{i=1}^{N_{p}} X_{i}(t)$ where $X_{i}(t)$ is the $x$-coordinate of particle, $i$, at time $t$ and $N_{p}$ is the number of particles. The $y$-coordinate of the plume centroid is: $\bar{Y}(t)=\frac{1}{N_{p}} \sum_{i=1}^{N_{p}} Y_{i}(t)$ where $Y_{i}(t)$ is the $y$-coordinate of particle, $\mathrm{i}$, at time $t$.

The variance around the centroid, which is a measure of plume spreading, is the second spatial moment around the mean which is computed as: $\sigma_{X X}^{2}(t)=\frac{1}{N_{p}} \sum_{i=1}^{N_{p}}\left[X_{i}(t)-\bar{X}(t)\right]^{2}$ for the longitudinal variance and $\sigma_{Y Y}^{2}(t)=\frac{1}{N_{p}} \sum_{i=1}^{N_{p}}\left[Y_{i}(t)-\bar{Y}(t)\right]^{2}$ for the transversal variance. The macrodispersion coefficients are derived from the longitudinal and transversal variances as $D_{x x}=\frac{1}{2} \frac{\partial \sigma_{X X}^{2}}{\partial t}$ and $D_{y y}=\frac{1}{2} \frac{\partial \sigma_{Y Y}^{2}}{\partial t}$ respectively (Dagan 1982).

\section{Generation of the heterogeneous medium}

The hydraulic conductivity is considered to be lognormally distributed (Smith 1981) with arithmetic mean
Fig. 2 Spatial moments of a pulse injection in a $2 \mathrm{D}$ flow field

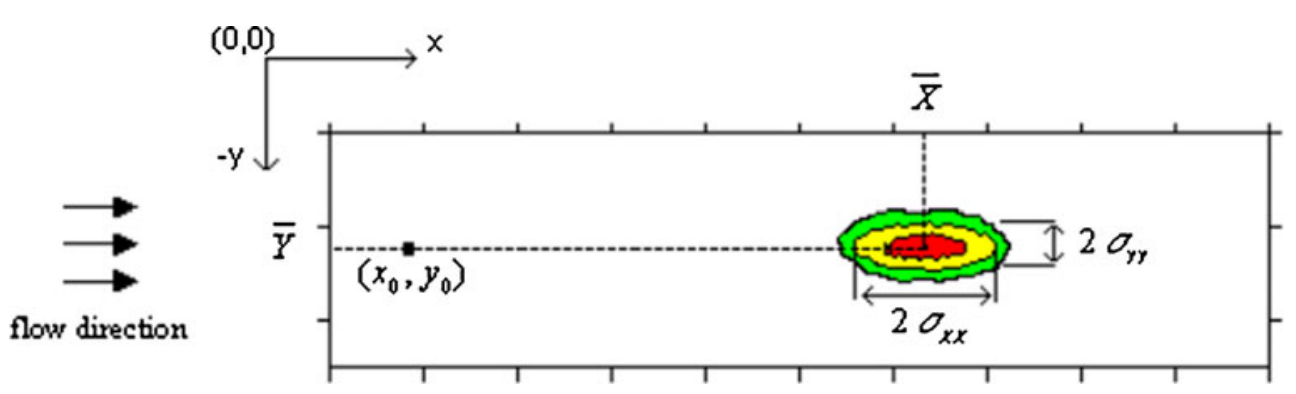


$\langle K\rangle$ and variance $\sigma^{2}{ }_{K}$. Therefore, the logarithmic transform $Y=\log (K)$ is normally distributed with mean $\langle Y\rangle$ and variance $\sigma^{2}{ }_{Y}$. The values of $\langle Y\rangle$ and $\sigma^{2}{ }_{Y}$ are related to $\langle K\rangle$ and $\sigma^{2}{ }_{K}$ through the logarithmic transformation

$$
\begin{aligned}
\langle Y\rangle & =\log \langle K\rangle-0.5 \sigma_{Y}^{2} \\
\sigma_{Y}^{2} & =\log \left(1+\frac{\sigma_{K}^{2}}{\langle K\rangle}\right)
\end{aligned}
$$

The corresponding harmonic mean $K_{\mathrm{h}}$ and geometric mean $K_{\mathrm{g}}$ of the lognormal distribution are given by

$$
\begin{aligned}
& K_{h}=\langle K\rangle e^{-\sigma_{Y}^{2}} \\
& K_{g}=\sqrt{K_{h}\langle K\rangle}
\end{aligned}
$$

The heterogeneous aquifer is modeled as a logGaussian random field characterized by a mean conductivity, $\langle K\rangle$, a standard deviation $\sigma_{K}$, and an exponential decay auto-correlation function characterized by a correlation length $\lambda$ (a measure of the distance which tells to what extent the values are correlated in space).

There are various methods to generate heterogeneous media (e.g. Haldorsen and Damsleth 1990, and Deutsch and Journel 1998). Comparison of some methods (e.g. multi-Gaussian method, nearest neighbor method and turning bands method) has been performed by Elfeki (2000).

Briefly, the turning bands method (Mantoglou and Wilson 1982) has some merits over other methods. It works by a fast generation of a stochastic process on a line and then projects the generated line process on the $2 \mathrm{D}$ or three-dimensional (3D) domain which is very cheap in terms of computer storage and speed. Most of the other methods work by building a huge matrix of the covariance function for all the points in the $2 \mathrm{D}$ or $3 \mathrm{D}$ domain and then decompose it with matrix operations. The turning bands method needs memory storage and time for matrix operations. Other methods such as the simulated annealing method, have the problem of being an iterative process where one needs to stop at certain criteria which might not be archived at the desired conversion.

Heterogeneous aquifers are generated using the program TBM2D developed by Elfeki (1996) based on the spectral turning bands method. The generation process on a random line is given by

$Z_{i}(u)=2 \sum_{j=1}^{M} \sqrt{\left[S_{1}\left(\omega_{j}\right) \cdot \Delta \omega\right]} \cos \left(\omega_{j}^{\prime} u+\phi_{j}\right)$

where $Z_{\mathrm{i}}(u)$ is a set of $N$ independent realizations of a onedimensional (1D), second-order stationarity stochastic process on a line $u, S_{1}\left(\omega_{\mathrm{j}}\right)$ is the spectral density function of the real process $Z(u)$ on the line, $\phi_{\mathrm{j}}$ represents independent random angles (which is uniformly distributed between 0 and $2 \pi$ ), $M$ is the number of harmonics used in the calculations, $\omega_{\mathrm{j}}=(j-0.5) \Delta \omega$, and $j=1,2, \ldots . \Delta . \Delta \omega$ is the discretized frequency (which is given by $\omega_{\max } / M$ ). $\omega_{\max }$ is the maximum frequency used in the calculations. $\omega_{\mathrm{j}}=\omega_{\mathrm{j}}+\delta \omega$, where $\delta \omega$ is a small random frequency added here in order to avoid periodicities. $\delta \omega$ is uniformly distributed between $-\Delta \omega^{\prime} / 2$ and $\Delta \omega^{\prime} / 2$, where $\Delta \omega^{\prime}$ is a small frequency such that $\Delta \omega<<\Delta \omega . \Delta \omega$ is taken equal to $\Delta \omega / 20$ according to Shinozuka and Jan (1972).

The construction of a simulated field $Z_{\mathrm{s}}(x, y)$ is performed by discrete simulation of the one-dimensional process $Z_{\mathrm{i}}(u)$ on a number of lines, $L$, and subsequent projection of the generated values onto all simulation points $(x, y)$ in the $2 \mathrm{D}$ domain. The random field is generated at each point according to

$Z_{S}(x, y)=\frac{1}{\sqrt{L}} \sum_{i=1}^{L} Z_{i}(u)$

\section{Model parameters of the numerical experiments}

The present study has considered various aquifer conditions and parameters to be investigated. Table 1 shows the parameters considered in the flow and transport experiments. The experiments have considered the influence of the size of the flow domain, the boundary conditions of the flow field, the parameters of the head fluctuation function (amplitude and period), the size of the source (initial condition) of the solute mass and the corresponding number of particles, the shape of the source, medium porosity, specific storage, and pore-scale longitudinal and lateral dispersivities. The model parameters cover the influence of correlation length of an isotropic medium that describes the size of the heterogeneity under ergodic and nonergodic conditions. The parameters also cover the influence of three degrees of heterogeneity.

Some common dimensionless numbers have been estimated to provide the limitations of application. The dimensionless parameter that relates advection and dispersion processes of the particles is called the Peclet number, $\mathrm{Pe}=\mathrm{v} \lambda / D_{\mathrm{xx}}$, where $\mathrm{v}$ is the absolute value of $\mathrm{a}$ typical velocity, $\lambda$ is a typical system length (in the present study this is the correlation length) and $D_{\mathrm{xx}}$ is the pore scale dispersion coefficient. Peclet numbers smaller than one, $\mathrm{Pe}<1$, imply dispersion dominated transport behavior, whereas transport characterized by Peclet numbers larger than one, $\mathrm{Pe}>1$, is dominated by advection.

Dentz and Carrera (2005) have used the non-dimensional Kubo number, $\kappa=\tau / \tau_{\mathrm{v}}$, which compares the correlation time of the flow fluctuations, $\tau$, to the advection time scale, $\tau_{\mathrm{v}}$. It equivalently compares the distance $\lambda_{\kappa}=\mathrm{V} \tau$, (Kubo distance) over which the solute is advected by the mean flow during one correlation time, $\tau$, to the correlation length in the direction of the mean flow, $\lambda,\left(\kappa=\lambda / \lambda_{\kappa}\right)$. The Kubo time scale is related to the dispersion time scale $\tau_{\mathrm{D}}$ by the relation $\tau_{\kappa}=(1+\kappa)$ 
Table 1 Simulation parameters used in computations

\begin{tabular}{ll}
\hline Parameter & Range of the numerical values \\
\hline Domain dimensions $(\mathrm{m})$ & Domain $500 \times 50$ and Domain $300 \times 50$ \\
Domain discretization $(\mathrm{m})$ & $1.0 \times 1.0$ \\
Time step (day) & 1.0 \\
Upstream fxed head boundary (m) & A COS $(\mathrm{i} .2 \pi \Delta t / P), \mathrm{i}=1,2, \ldots, 50$ \\
Downstream head boundary (m) & $A=1,10,20 \mathrm{~m}$ \\
& 40 and 20 \\
Period of oscillation (days) & 10 \\
Constant aquifer thickness (m) & 10 \\
Mean hydraulic conductivity (m/day) & $1,5,10$ \\
Standard deviation in hydraulic conductivity (m/day) & 0.001 \\
Accuracy in computation & 50 \\
No. of time steps & $0.001,0.01,0.1$ \\
Storage coefficient & 0.4 \\
Porosity & $1,2,3$ and $1,2,5,15,25$ \\
Correlation lengths (m) & Point-like source (one cell size) $1 \times 1$ \\
Source size (m) & Line-like source (horizontal $\times$ vertical) $1 \times 50$ \\
& $\mathrm{x}_{\mathrm{o}}=10$ and $\mathrm{y}_{\mathrm{o}}=-25$ \\
Source centroid location (m) & $0.1,0.5$ \\
Longitudinal pore scale dispersivity (m) & $0.01,0.05$ \\
Transverse pore scale dispersivity (m) & 100,000 \\
No. of particles & 25,000 \\
Injected solute mass (grams) &
\end{tabular}

$\tau_{\mathrm{D}}$ (Dentz and Carrera 2005). The values of the dimensionless parameters are presented in Table 2.

\section{Analysis of the results}

\section{Influence of the correlation length (order of the heterogeneity)}

Heterogeneous aquifers are generated using the same mean and standard deviation of the hydraulic conductivity, and with different correlation lengths. As a rule of thumb, the plume, under steady-state flow conditions, should travel at least 20-25 correlation lengths to reach asymptotic behavior (Ababou et al. 1989). The same condition has been considered for the unsteady state within one cycle of the oscillation. As a result, in one cycle the plume travels a distance large enough to discover all heterogeneities in the aquifer. Thus, in the next cycle, the plume will discover the same heterogeneities and therefore heterogeneity is no longer contributing to the spreading. With the boundary conditions imposed, the plume travels approximately $40 \mathrm{~m}$ in one cycle of 40 days. So simulations in aquifers are only done with correlation lengths 1,2 and $3 \mathrm{~m}$. Moreover, this study takes a source that covers the whole width of the aquifer $(1 \mathrm{~m} \times 50 \mathrm{~m})$. Indeed, if a point-like source $(1 \mathrm{~m} \times 1 \mathrm{~m})$ is used, the plume is small with respect to the size of heterogeneities and follows only one path and will not discover all heterogeneities unless the travel time tends to infinity. Then, a large source enables one to sample all the heterogeneities in the aquifer and the plume becomes ergodic.

The centroid displacement (Fig. 3) shows that the plume moves slower when the correlation length increases. This is due to the tortuosity of the particle paths which increases with the correlation length; the plume moves around zones of low conductivity which leads to longer paths when the correlation length is long. The longitudinal variance and macrodispersion coefficient show larger variation in the spreading when the correlation length increases.

The plume evolution for correlation length $3 \mathrm{~m}$ (Fig. 4) enables one to see that, indeed, some part of the plume stays behind in the zone of low conductivity while another part goes ahead in the zone of high conductivity; a phenomenon of channelling appears when the correlation length increases. On the other hand, the variance in $x$ direction can go down as shown at correlation length of $3 \mathrm{~m}$ after 200 days since release. The reason is that the plume may face the zone of low conductivity which makes the front of the plume slow down, while the tail of the plume may be located in the high conductivity zone which makes the tail move faster. This causes the plume to shrink and therefore causes the variance to decrease. This

Table 2 Parameters estimated from the simulations

\begin{tabular}{|c|c|c|c|c|c|c|c|}
\hline$\lambda(\mathrm{m})$ & $\mathrm{v}$ (m/day) & $\tau_{\mathrm{v}}$ (days) & $\tau_{\mathrm{K}}$ (days) & $\tau_{\mathrm{D}}($ days $)$ & Period, $\tau$ (days) & Kubo No. & Peclet No. \\
\hline 1 & 1.6 & 0.6 & $25,606.3$ & 6.3 & 40 & 64 & 10 \\
\hline 2 & 1.4 & 1.4 & $22,428.6$ & 28.6 & 40 & 28 & 20 \\
\hline 3 & 1 & 3 & $16,010.1$ & 90.0 & 40 & 13.3 & 30 \\
\hline 1 & 1.5 & 0.7 & $6,006.7$ & 6.7 & 20 & 30 & 10 \\
\hline 5 & 1.5 & 3.3 & $6,166.7$ & 166.7 & 20 & 6 & 50 \\
\hline 15 & 1.5 & 10 & $7,500.0$ & $1,500.0$ & 20 & 2 & 150 \\
\hline 25 & 1.5 & 16.7 & $10,166.7$ & $4,166.7$ & 20 & 1.2 & 250 \\
\hline
\end{tabular}


Fig. 3 Spatial moments and macrodispersion coefficients of the plume for different correlation lengths 1,2 and 3 $\mathrm{m}$. Aquifer characteristics length $=500 \mathrm{~m},\langle K\rangle=10 \mathrm{~m} /$ day, $\sigma_{K}=5 \mathrm{~m} /$ day, $\mathrm{S}=0.001, \alpha_{L}=$ $0.1 \mathrm{~m}, \alpha_{T}=0.01 \mathrm{~m}$. The downstream water level fluctuates with a period of 40 days and amplitude of $20 \mathrm{~m}$. The source size is $1 \mathrm{~m} \times 50 \mathrm{~m}$
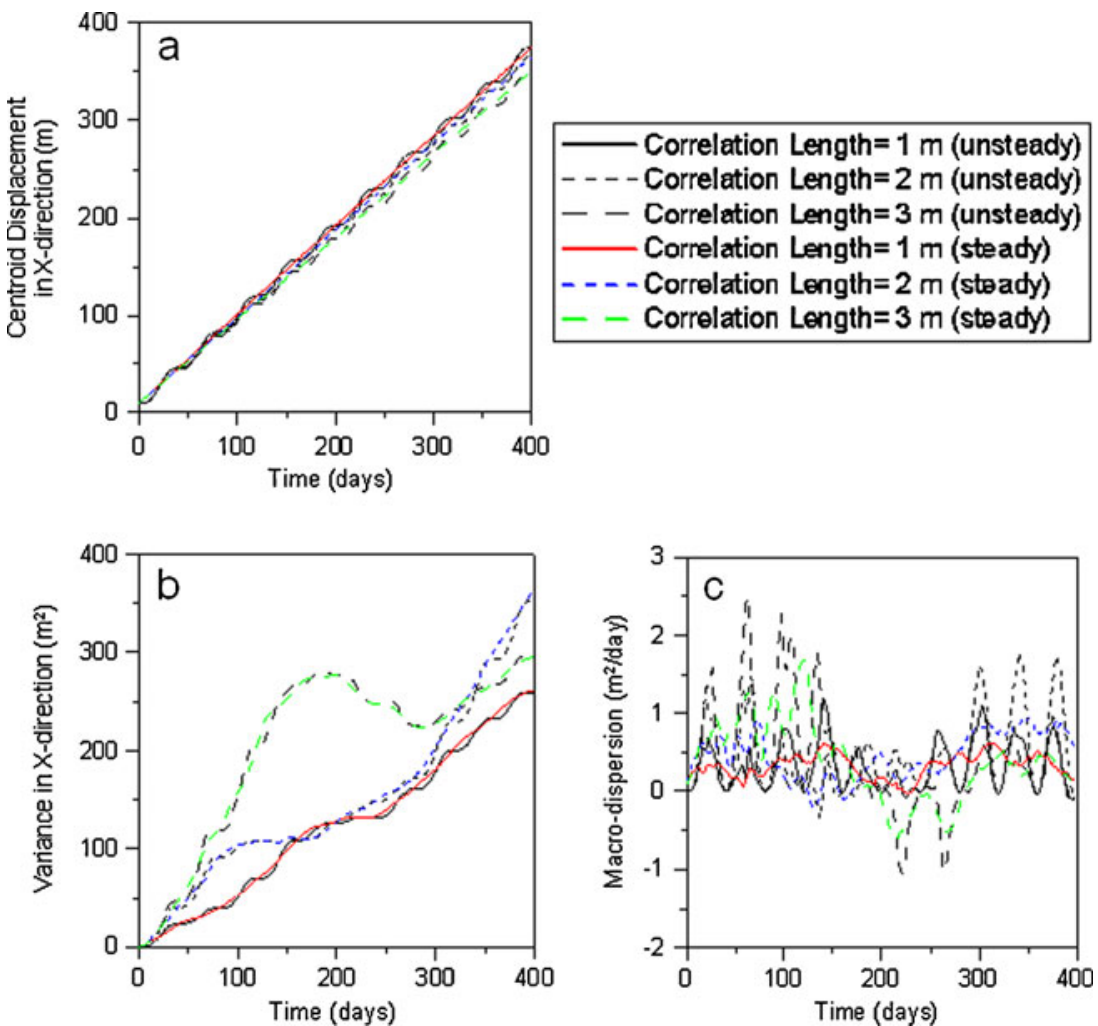

feature could lead to a negative macrodispersion as shown in Fig. 3. Some studies have shown this behavior. The reason behind this behavior, in the case of spatial variability, is that when there are diverging flow lines, the front of the plume moves slower than the tail of the plume so that the plume shrinks in the longitudinal direction leading to a decrease in the longitudinal variance and consequently a negative macrodispersion (Elfeki et al. 1996, 1997, 2007). Negative dispersion has also been reported in the literature in coastal water where oscillating unsteady-state flow conditions are present (List et al. 1990).

The main observation concerning transient conditions is that results under transient conditions oscillate around
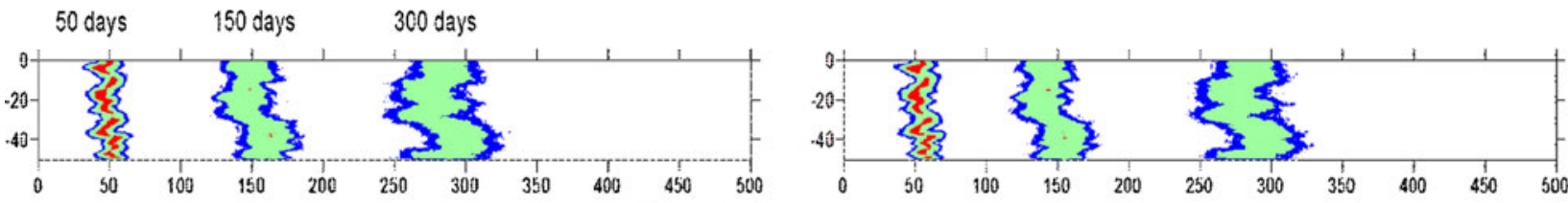

Correlation Length $=1 \mathrm{~m}$
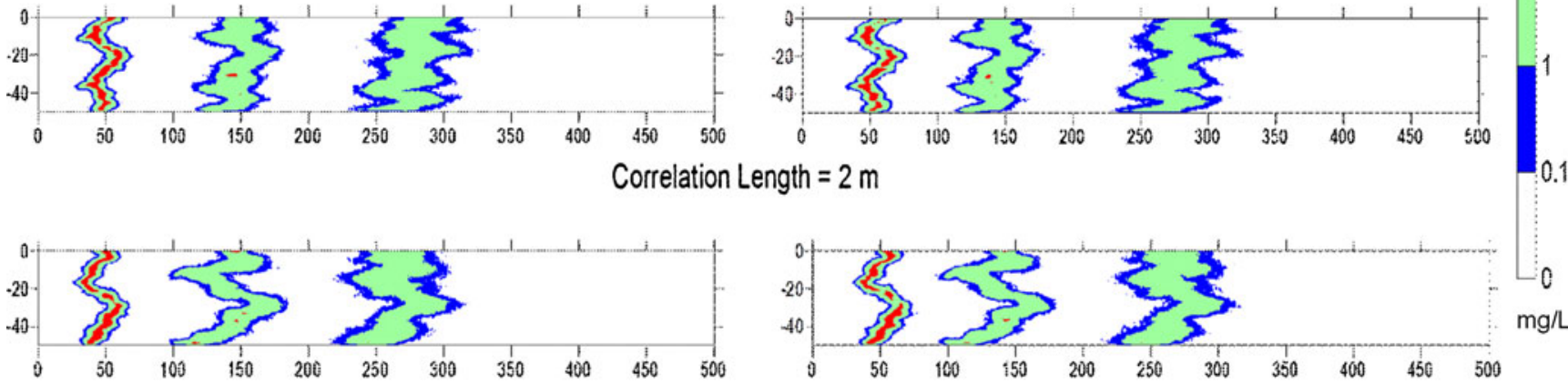

Correlation Length $=2 \mathrm{~m}$

Unsteady Conditions

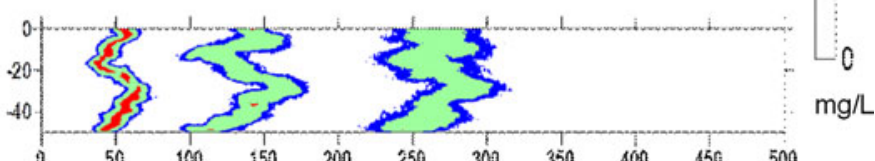

Correlation Length $=3 \mathrm{~m}$

Fig. 4 Plume evolution in three aquifers of correlation lengths 1,2 and $3 \mathrm{~m}$. Aquifer characteristics: length $=500 \mathrm{~m},\langle K\rangle=10 \mathrm{~m} /$ day, $\sigma_{K}=5$ $\mathrm{m} /$ day, $S=0.001, \alpha_{L}=0.1 \mathrm{~m}, \alpha_{T}=0.01 \mathrm{~m}$. The downstream water level fluctuates with a period of 40 days and amplitude of $20 \mathrm{~m}$. The source size is $1 \mathrm{~m} \times 50 \mathrm{~m}$ (Concentration scale is in $\mathrm{mg} / \mathrm{L}$ and dimensions in meters) 
the steady-state case. The difference between both states is relatively small. The evolution and shape of the plume under transient condition is similar to the steady-state case (Fig. 4).

Figure $5 \mathrm{a}$ and $\mathrm{b}$ show transport spatial moments performed in aquifers with correlation lengths which do not fulfil the condition 'distance travelled $>20 \lambda$ '. The distance travelled within one cycle is about $30 \mathrm{~m}$, thus for aquifers with $\lambda=5,15$ and $25 \mathrm{~m}$, the ergodic condition is not fulfilled. Therefore, there is no trend obtained in the results when $\lambda$ increases; however, periodicity is presented in the results. Figure $5 \mathrm{c}$ and $\mathrm{d}$ show the macrodispersion in both longitudinal $x$-direction and transverse y-direction. Longitudinal macrodispersion shows periodicity; however, no regular pattern can be observed due to the increase in the correlation length. For transverse macrodispersion, periodicity is not observed clearly due to the fact that the sources are extended laterally over the whole domain.

\section{Influence of the standard deviation}

Several heterogeneous aquifers have been generated. All have the same correlation length and the same arithmetic mean, but the standard deviation is variable. Increasing the standard deviation means that the range of hydraulic conductivity values is wider. Because the lognormal distribution (i.e. asymmetrical distribution) is used, generated hydraulic conductivity values that are lower than the arithmetic mean $(10 \mathrm{~m} /$ day $)$ are more likely to be present in the medium, compared with those above the arithmetic mean (Fig. 6).

The transport simulation has been performed for both steady and unsteady flow with a source covering all the width of the aquifer and a downstream level fluctuating with a period of 40 days and amplitude of $20 \mathrm{~m}$. Figures 7 and $8 \mathrm{a}$ show that the plume travels slower for high

Fig. 5 Spatial moments and macrodispersion under non-ergodic conditions. a centroid displacement in $\mathrm{x}$-direction, $\mathbf{b}$ Variance in $\mathrm{x}$-direction, $\mathbf{c}$ Longitudinal macrodispersion, and d Transversal macrodispersion. Aquifer characteristics: length $=300 \mathrm{~m},\langle K\rangle=10$ $\mathrm{m} /$ day, $\sigma_{K}=5 \mathrm{~m} /$ day, $S=0.001$. The downstream water level fluctuates as a cosine function with a period of 20 days and amplitude of $20 \mathrm{~m}$. The source size is $1 \mathrm{~m} \times 50 \mathrm{~m}$ standard deviation because there are more zones of low conductivity generated in the field when the standard deviation is increased.

Figure $8 \mathrm{~b}$ shows the longitudinal $\mathrm{x}$-direction variance of the plume for both steady and unsteady conditions. The spreading of the plume is enhanced in the longitudinal direction with increasing standard deviation. The spreading increases because the contrast in hydraulic conductivity values is higher in the case of high standard deviation. In the macrodispersion coefficient plots (Fig. 8c and d), periodic variations are obvious with increasing amplitude due to the increase in the standard deviation of the hydraulic conductivity. Periodicity is present in both the variance and macrodispersion coefficient. It is also noticed that the variation in both the longitudinal and transverse macrodispersion coefficients are sharper in the case of unsteady flow when compared with steady flow conditions. This behavior is due to the fact that under unsteady flow conditions, parts of the plume might be stagnant while other parts might be moving, so the plume is shrunk and stretched suddenly, which leads to this sharpening in the variation of the macrodispersion coefficients. The magnitude of the longitudinal macrodispersion coefficient under unsteady flow condition is almost doubled when compared with the steady-state flow condition. However, the transverse macrodispersion coefficient fluctuates around zero for the whole period covered (ie. along the horizontal axis).

\section{Influence of the magnitude of the amplitude}

Figure 9 displays plume spatial moments and macrodispersion under three values of wave amplitude $(A=1,10$, and $20 \mathrm{~m}$ ) at the upstream boundary. It is obvious, from the first moment (plume centroid), that the plume moves the slowest at the lowest amplitude, $1 \mathrm{~m}$, and the fastest at
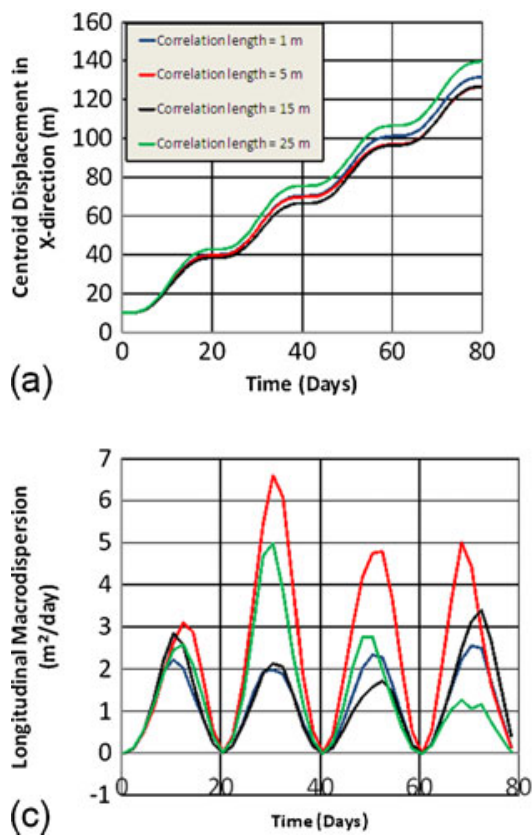
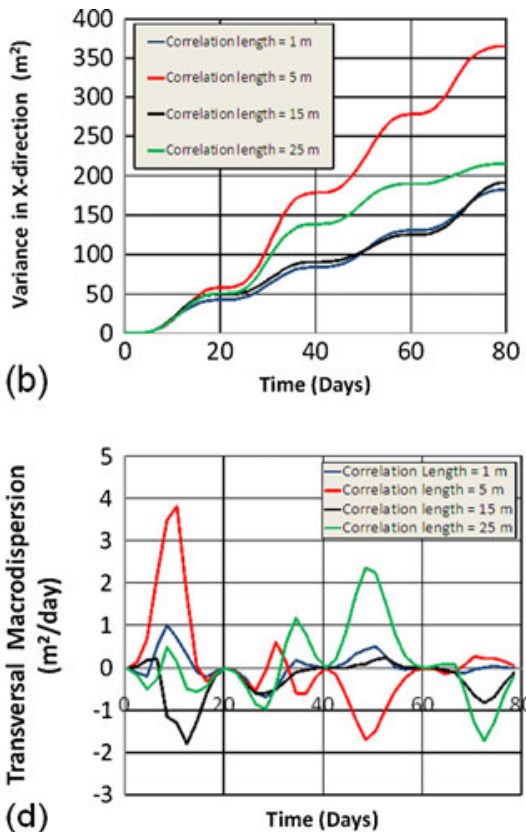

(d)

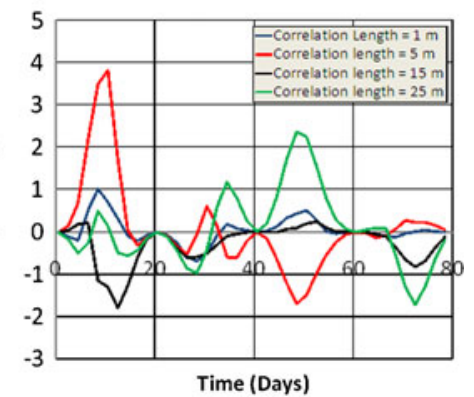



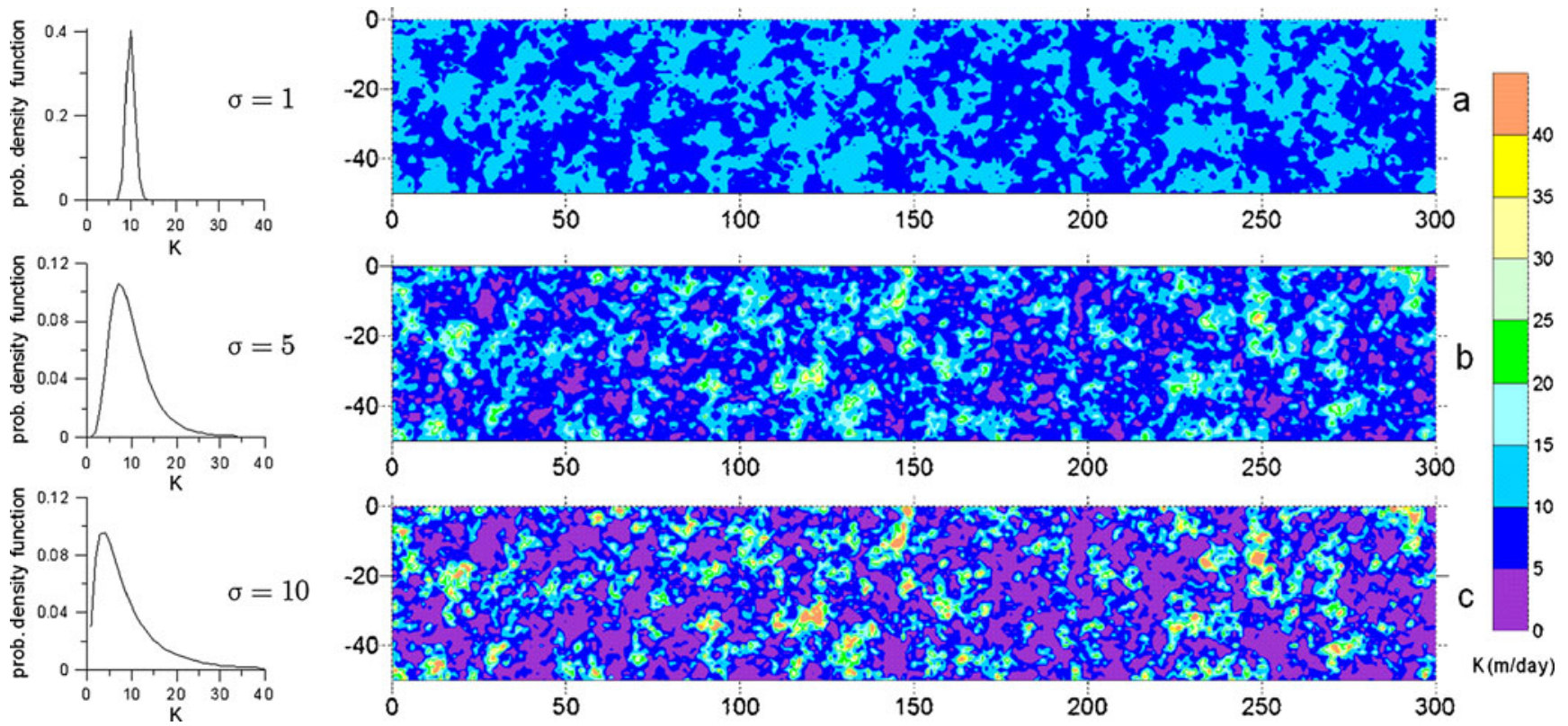

Fig. 6 Hydraulic conductivity $(K)$ distribution $(\mathrm{m} /$ day) in heterogeneous media with the same correlation length of $2 \mathrm{~m}$ and arithmetic mean $10 \mathrm{~m} /$ day, but different standard deviations: $\sigma_{K}=1,5$ and $10 \mathrm{~m} /$ day. Domain dimensions are in meters

the highest amplitude, $20 \mathrm{~m}$. This is due to the high gradients induced by high amplitudes. The middle value, $10 \mathrm{~m}$, lies in between the two extremes. The second longitudinal spatial moment (longitudinal variance) shows increase in the variance with increase in the wave amplitude. This leads to the largest spreading of the plume at the $20-\mathrm{m}$ amplitude. The longitudinal macrodispersion coefficient displays oscillatory changes due to the wave fluctuations at the boundary. The appearance of negative macrodispersion is due to the instantaneous extension and reduction of the longitudinal variance of the plume. The variability in the macrodispersion coefficient increases with the increase of the wave amplitude that is reflected by the high gradients. The same behavior has been also observed in homogenous media (Elfeki et al. 2007).

\section{Influence of the storage coefficient}

Figure 10 shows the effect of the storage coefficient on the plume spatial moments and the longitudinal macrodispersion. It is obvious that the increase in the storage coefficient leads to a decrease in the spatial moments and longitudinal macrodispersion. In the case of the high storage coefficient value $(S=0.1)$, the wave is damped in the aquifer and the aquifer response is delayed. However, in the case of the lowest storage coefficient value $(S=$ $0.001)$, the immediate aquifer response is manifested on the spatial moments and the longitudinal macrodispersion where the plume reaches a steady oscillations. The middle value case $(S=0.01)$ shows the combined effects of both the aforementioned extreme cases (i.e. there is a delay effect in the beginning, and the immediate response is reached after about 100 days). The longitudinal macro-
Fig. 7 Plume evolution in heterogeneous aquifers with the same correlation length of $2 \mathrm{~m}$ but with different standard deviations of hydraulic conductivity. Aquifer characteristics: length $=300 \mathrm{~m},\langle K\rangle=10$ $\mathrm{m} /$ day, $\lambda_{\mathrm{x}}=\lambda_{\mathrm{y}}=2 \mathrm{~m}, S=0.001$. The downstream water level fluctuates with a period of 40 days and amplitude of $20 \mathrm{~m}$. Strip source of contamination. Domain dimensions are in meters
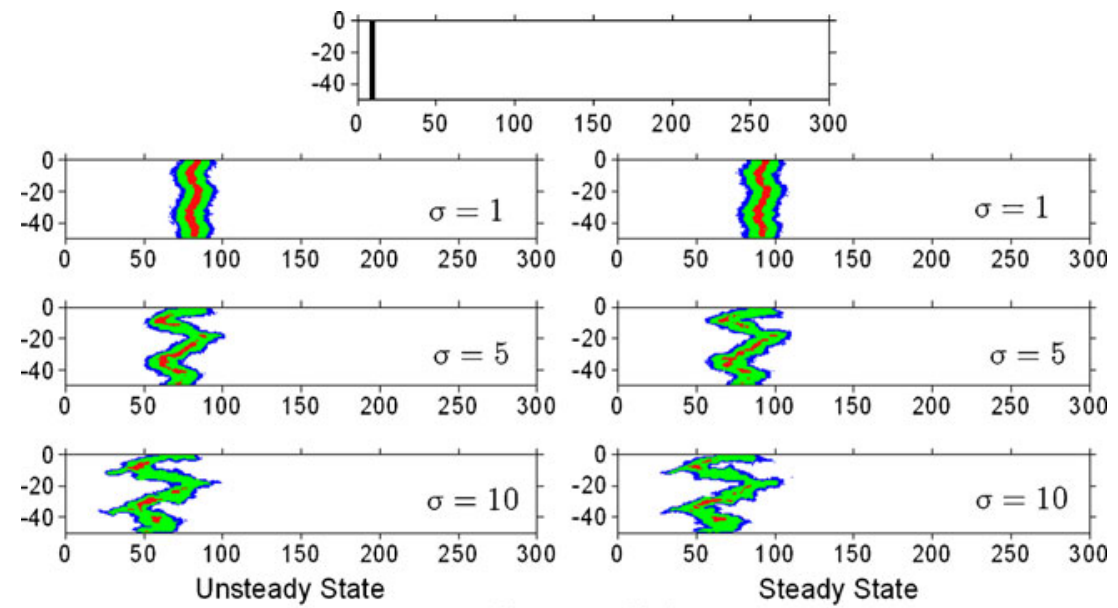

Plumes at 50 days

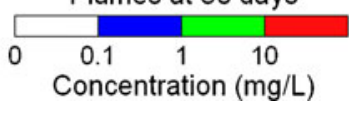


Fig. 8 Plume spatial moments and macrodispersion in heterogeneous aquifers with the same correlation length of $2 \mathrm{~m}$ but with different standard deviations of hydraulic conductivity $(S D)$. a Centroid displacement in $\mathbf{x}$-direction, $\mathbf{b}$ Variance in $\mathrm{x}$-direction, $\mathbf{c}$ Longitudinal macrodispersion, d Transversal macrodispersion. Aquifer characteristics: length $=300 \mathrm{~m},\langle K\rangle=10 \mathrm{~m} /$ day, $\lambda_{\mathrm{x}}=\lambda_{\mathrm{y}}=2 \mathrm{~m}, S=0.001$. The downstream water level fluctuates with a period of 40 days and amplitude of $20 \mathrm{~m}$. Strip source of contamination
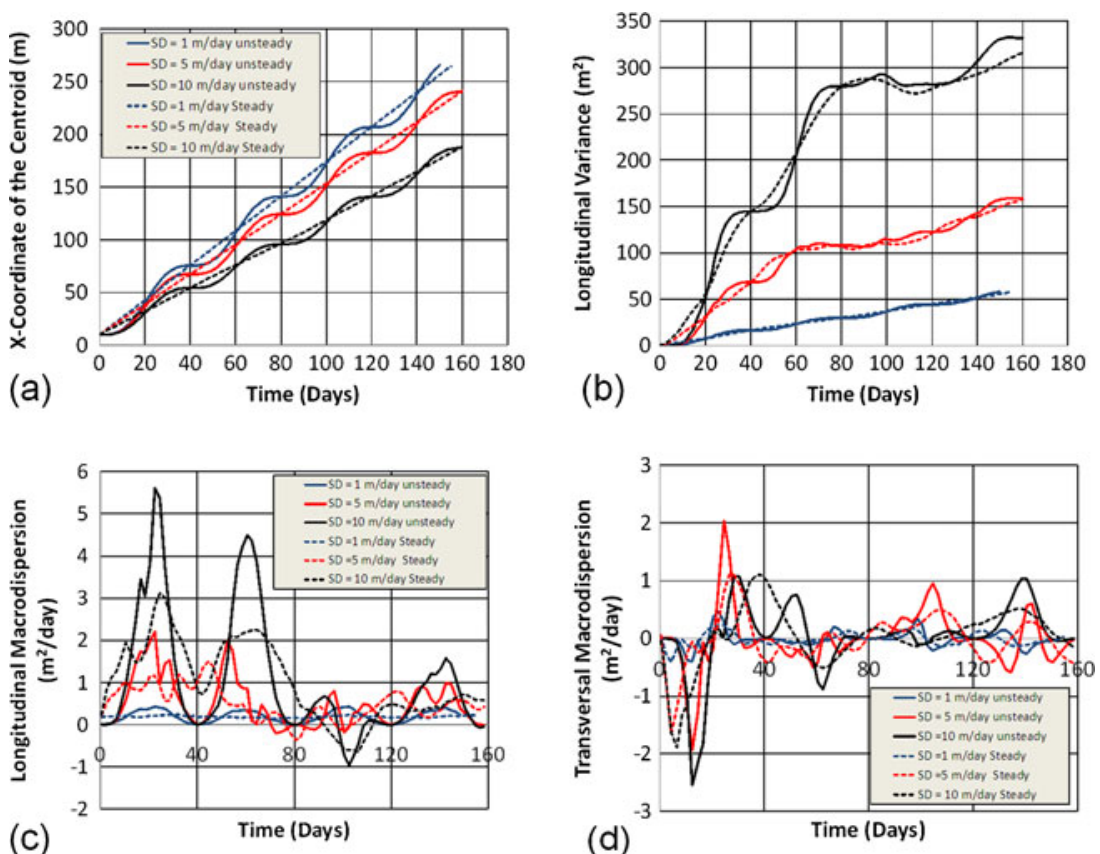

dispersion is getting amplified especially for the middle case because this case shows the plume development until it reaches a state of steady oscillations.

\section{Influence of pore-scale dispersivity}

Figure 11 presents the effect of pore-scale dispersivity on the plume spatial moments and longitudinal macrodispersion. Two cases are considered, where longitudinal dispersivities are 0.1 and 0.5 , and where the transverse dispersivities are taken as one tenth of the longitudinal ones. The first moment is not presented because the mean velocity is not influenced by the pore-scale dispersivities. However, the second moment has been presented showing high spreading with high pore-scale dispersivity, since it enhances spreading. The variability of the longitudinal macrodispersion increases with larger value of pore-scale dispersivity and the amplification of the longitudinal macrodispersion appears as the plume reaches the fluctuating boundary.

\section{Spreading due to heterogeneity and transient flow}

In heterogeneous aquifers, the spreading is caused by the spatial variation in the hydraulic conductivity values on
Fig. 9 Plume spatial moments and longitudinal macrodispersion in heterogeneous aquifers with the same correlation length of $2 \mathrm{~m}$ but with different amplitude $(A)$ at the downstream boundary. a Centroid displacement in $\mathrm{x}-$ direction, $\mathbf{b}$ Variance in $\mathrm{x}-$ direction, c Longitudinal macrodispersion. Aquifer characteristics: length $=300 \mathrm{~m},\langle K\rangle=$ $10 \mathrm{~m} /$ day, $\lambda_{\mathrm{x}}=\lambda_{\mathrm{y}}=2 \mathrm{~m}, S=$ 0.001 . The downstream water level fluctuates with a period of 40 days. Strip source of contamination
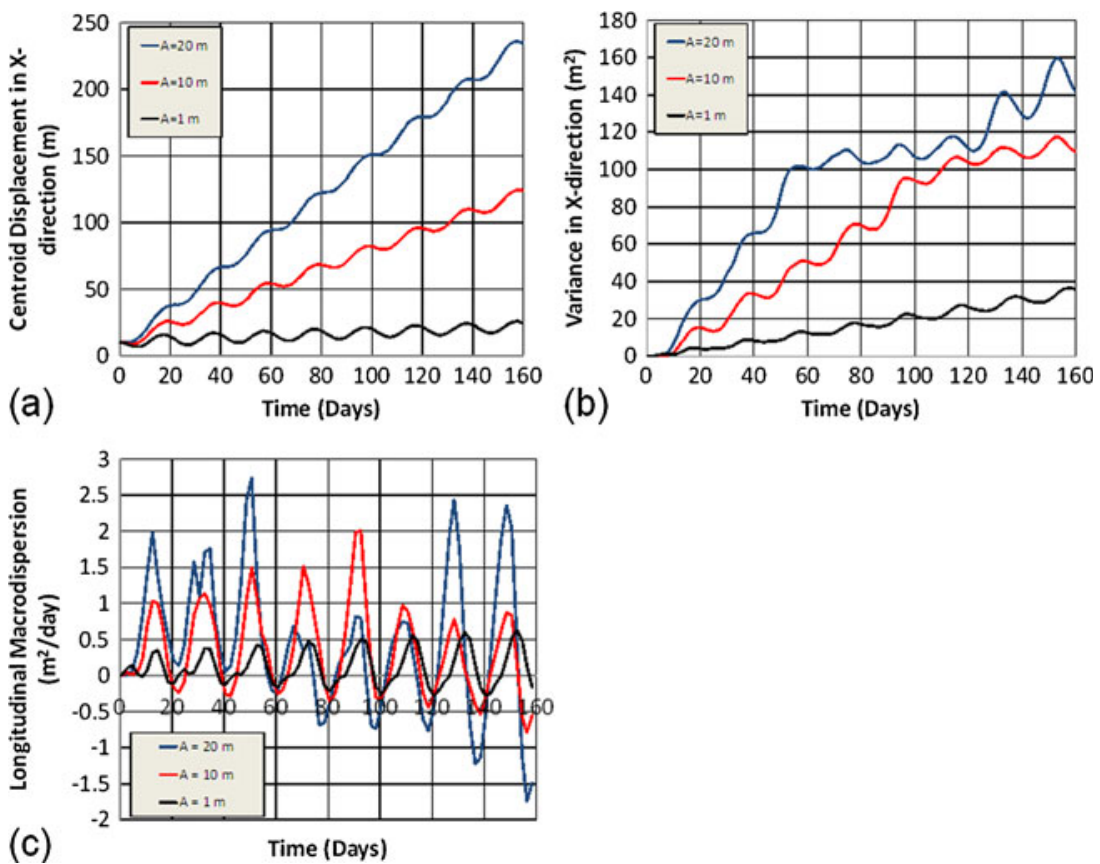
Fig. 10 Plume spatial moments and longitudinal macrodispersion in heterogeneous aquifers with the same correlation length of $2 \mathrm{~m}$ but with different storage coefficients $(S=0.001,0.01,0.1)$. a Centroid displacement in $\mathrm{x}$ direction, $\mathbf{b}$ Variance in $\mathrm{x}-$ direction, and c Longitudinal macrodispersion. Aquifer characteristics: length $=300 \mathrm{~m}$, $\langle K\rangle=10 \mathrm{~m} /$ day, $\lambda_{\mathrm{x}}=\lambda_{\mathrm{y}}=2 \mathrm{~m}$. The downstream water level fluctuates with a period of 40 days. Strip source of contamination
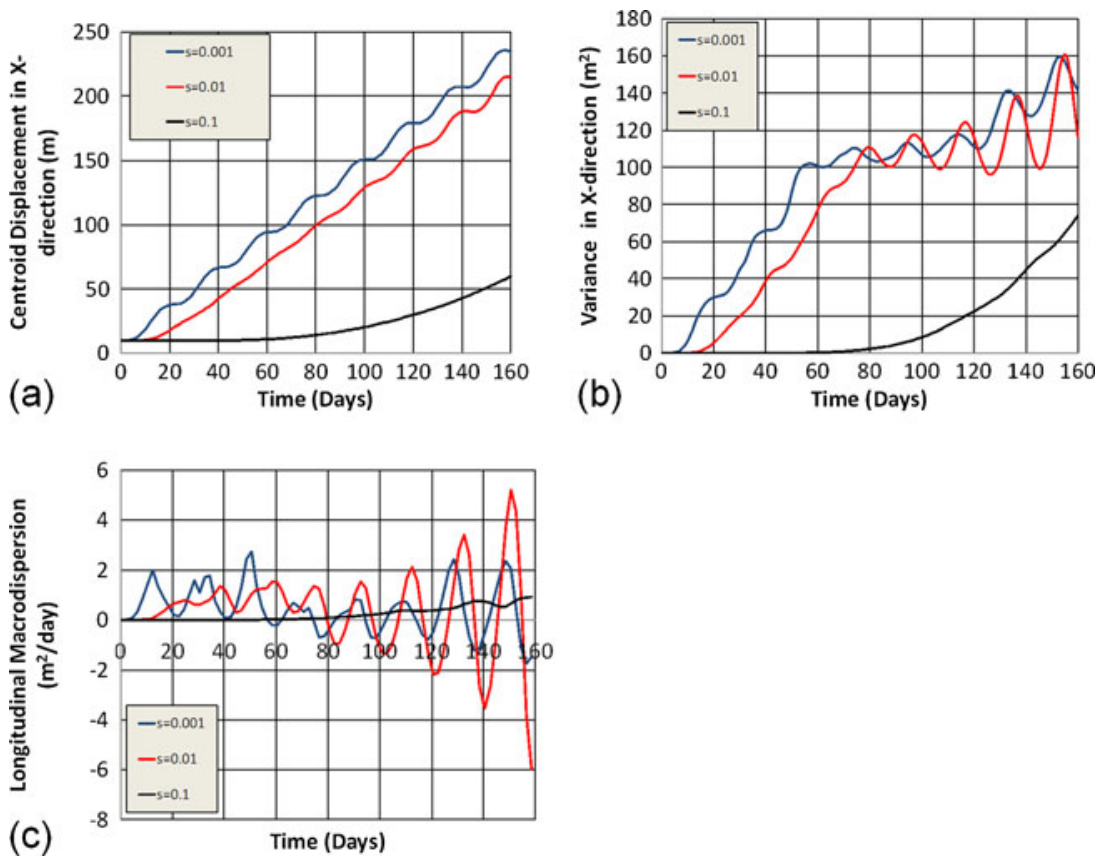

one hand and by the temporal variations in the head field on the other hand. However, in the steady-state case, the spreading is only due to spatial variations. Therefore it was expected that if the steady component was subtracted from the transient component, only the component due to temporal fluctuations would remain (see Fig. 12). A pointlike source of contaminant was injected in a heterogeneous aquifer. The transport is simulated under steadystate conditions and also under transient conditions in both homogeneous (with the mean hydraulic conductivity) and heterogeneous media (the downstream level is a cosine function with a period of 20 days and amplitude of $20 \mathrm{~m}$ ). The results are presented in Figs. 13 and 14, for homogenous and heterogeneous media respectively, which shows that the temporal component is still periodic in the heterogeneous media, Fig. 14, but is no longer a perfect sine like in the homogenous aquifer (Fig. 13).

It is difficult to interpret, but it seems that spreading is not simply a component due to temporal fluctuations added to a component due to heterogeneity (otherwise the residual would have a symmetric shape). It seems, rather, that spatial and temporal variations interact in a complex manner to produce variations in the velocity field and thus in the plume spreading.

\section{Conclusions}

Simulations of solute transport in heterogeneous porous media show that the effect of temporal variations on the transport of solutes is masked by the strong effect of spatial heterogeneity. There is no obvious difference in terms of plume shape between steady and unsteady flow conditions; however, the difference is obvious in the first moment, second moment and macrodispersion coefficients. The spatial moments of the plume of the unsteady-state flow condition fluctuate around the steady-state flow condition. The period of oscillations in the flow field is reflected in plume spatial moments and macrodispersion coefficient signals when the storage
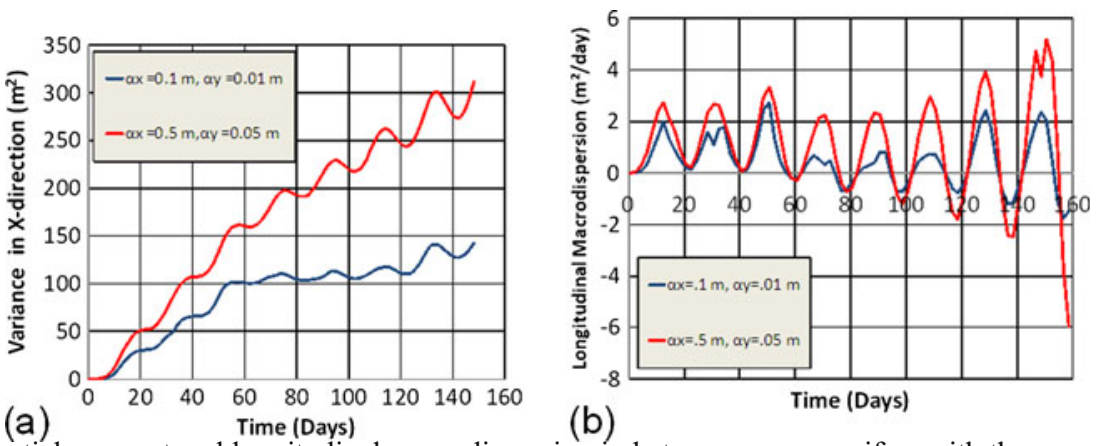

(b)

Fig. 11 Plume second spatial moment and longitudinal macrodispersion in heterogeneous aquifers with the same correlation length of $2 \mathrm{~m}$ but with different longitudinal dispersivities $\left(a_{x}\right)$ of 0.1 and 0.5 and transverse dispersivities $\left(a_{y}\right)$ of 0.01 and $0.05 \mathrm{~m}$. a Variance in $\mathrm{x}-$ direction, and b Longitudinal macrodispersion. Aquifer characteristics: length $=300 \mathrm{~m},\langle K\rangle=10 \mathrm{~m} /$ day, $\lambda_{x}=\lambda_{y}=2 \mathrm{~m}$. The downstream water level fluctuates with a period of 40 days. Strip source of contamination 

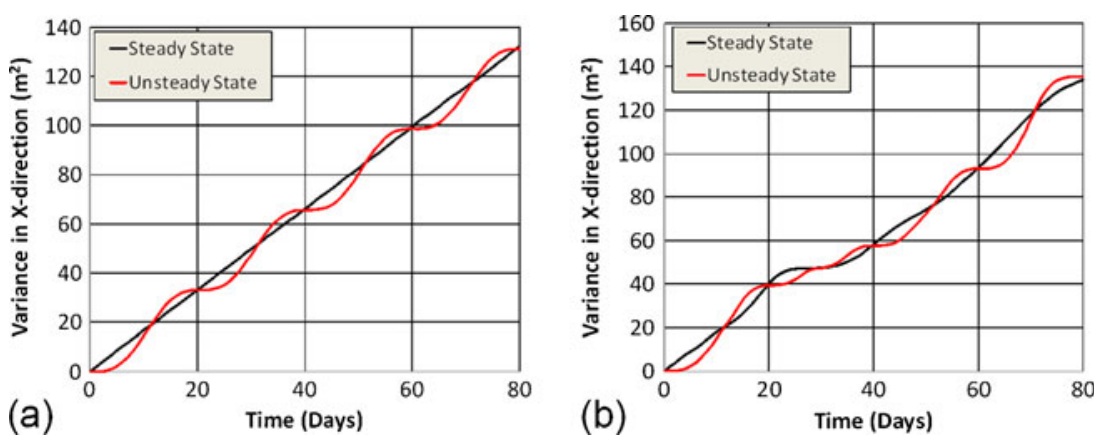

Fig. 12 Comparison between longitudinal variance in case of steady and unsteady states in a a homogenous aquifer and $\mathbf{b}$ heterogeneous aquifer. Aquifer characteristics: $S=0.001 ; K=10 \mathrm{~m} /$ day in homogeneous case; $\langle K\rangle=10 \mathrm{~m} /$ day, $\sigma_{K}=5 \mathrm{~m} /$ day and $\lambda_{\mathrm{x}}=2 \mathrm{~m}$ in heterogeneous case. The downstream water level fluctuates with a period of 20 days and an amplitude of $20 \mathrm{~m}$. Point source of contamination

coefficients are small $(S \leq 0.001)$. At a relatively high degree of the standard deviation in hydraulic conductivity and at a small storage coefficient, the unsteady flow condition sharpens the temporal variations in the macrodispersion coefficients. The magnitude of the longitudinal macrodispersion coefficient under unsteady flow condition is almost doubled at the maximum values when compared with the steady-state flow condition. However, for the transverse macrodispersion coefficient, the magnitude fluctuates around zero, i.e. along the horizontal axis, under the range of parameters used in this study. The presented simulations have covered the Kubo number range of 1.2-64, while it covers the Peclet number range of 10-250. The spatial moments of the plume show that spatial and temporal variations interact together in a complex manner to produce variations in the velocity field, and therefore it is difficult to distinguish the influence of transient conditions from the influence of heterogeneity on the transport of solutes. This conclusion is supported by other researchers (Rehfledt and Gelhar 1992; Dagan et al. 1996) who concluded that heterogene- ity and time variation effects cannot be treated separately. The numerical experiments of this study have emphasized that the plume spreading increases with increasing $\sigma_{K}$. The temporal variations in the macrodispersion coefficients are increasing when the contrast in the hydraulic conductivity is high.

\section{Appendix A: derivation of the numerical groundwater flow model}

A numerical scheme with a five-point operator is used. The solution is based on the backward difference approximation in order to get a stable solution whatever the size of the time step. The following equation must then be transformed

$$
S \frac{h_{i, j}^{k+1}-h_{i, j}^{k}}{\Delta t}=\frac{\partial}{\partial x}\left(T_{x x} \frac{\partial h^{k+1}}{\partial x}\right)+\frac{\partial}{\partial y}\left(T_{y y} \frac{\partial h^{k+1}}{\partial y}\right)
$$

Fig. 13 Difference between steady and unsteady state in a homogeneous aquifer. a Difference of centroid displacement in x-direction between unsteady and steady flow conditions, b Difference of variance in x-direction between unsteady and steady flow conditions, and $\mathbf{c}$ Difference of variance in y-direction between unsteady and steady flow conditions. Aquifer characteristics: $K=10 \mathrm{~m} /$ day and $S=0.001$. Point source of contamination
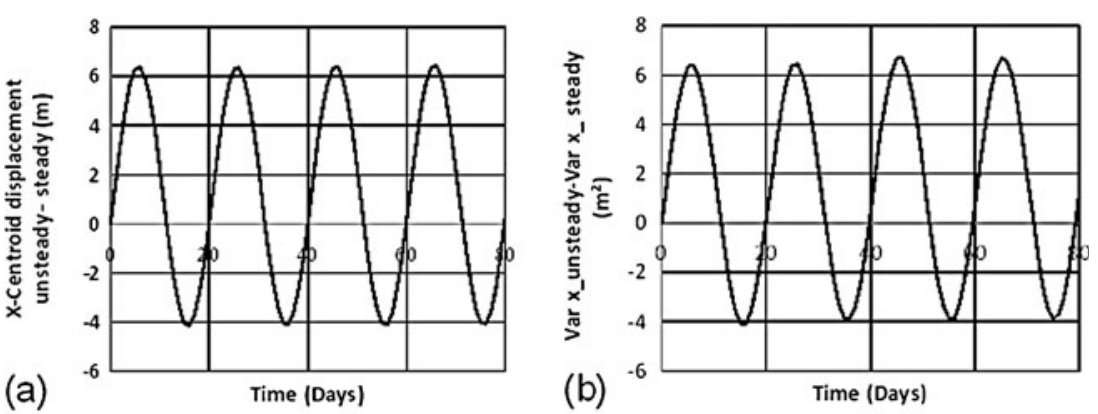
Fig. 14 Difference between steady and unsteady state in a heterogeneous aquifer. a Difference of centroid displacement in $\mathrm{x}$-direction between unsteady and steady flow conditions, b Difference of variance in $\mathrm{x}$-direction between unsteady and steady flow conditions, and c Difference of variance in $y$-direction between unsteady and steady flow conditions. Aquifer characteristics: $S=$ 0.001, $\langle K\rangle=10 \mathrm{~m} /$ day, $\sigma_{K}=5$ $\mathrm{m} /$ day and $\lambda_{\mathrm{x}}=2 \mathrm{~m}$. Point source of contamination
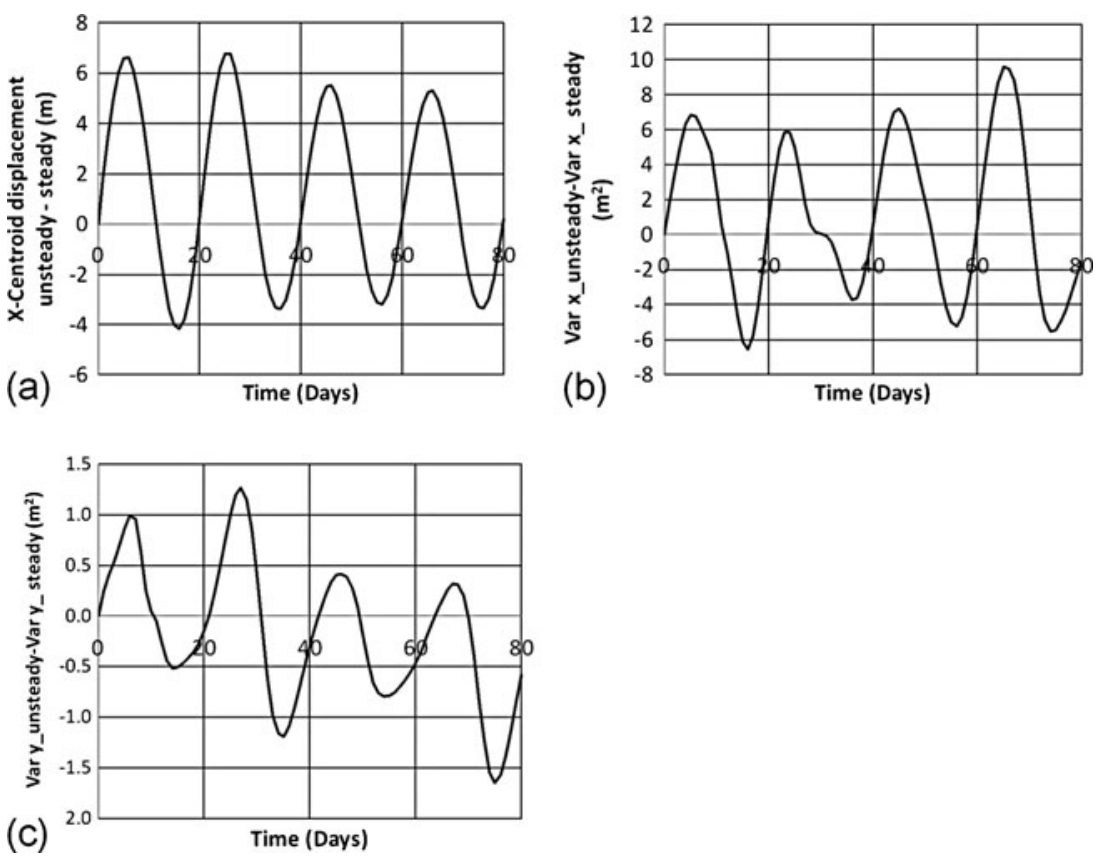

The finite difference analogue of the derivatives is with given by:

$T_{x x}(x, y) \frac{\partial h^{k+1}}{\partial x} \approx T_{x x_{i+1 / 2, j}}\left(\frac{h_{i+1, j}^{k+1}-h_{i, j}^{k+1}}{\Delta x}\right)$

with the harmonic mean of the transmissivity between neighboring nodes as $T_{x x_{i+1 / 2, j}}=\frac{2 T_{x x_{i+1, j}} T_{x x_{i, j}}}{T_{x x_{i+1, j}}+T_{x x_{i, j}}}$ and $h_{i, j}^{k+1}$ is the hydraulic head at the node $(i, j)$, at time $(k+1)$.

The derivative analogue with respect to $y$, is obtained similarly. Further evaluation leads to the following expressions of the second derivatives

$\frac{\partial}{\partial x}\left(T_{x x}(x, y) \frac{\partial h^{k+1}}{\partial x}\right) \approx \frac{T_{x x_{i+1 / 2, j}}\left(\frac{h_{i+1, j}^{k+1}-h_{i, j}^{k+1}}{\Delta x}\right)-T_{x x_{i-1 / 2, j}}\left(\frac{h_{i, j}^{k+1}-h_{i-1, j}^{k+1}}{\Delta x}\right)}{\Delta x}$

$\frac{\partial}{\partial y}\left(T_{y y}(x, y) \frac{\partial h^{k+1}}{\partial y}\right) \approx \frac{T_{y y_{i, j+1 / 2}}\left(\frac{h_{i, j+1}^{k+1}-h_{i, j}^{k+1}}{\Delta y}\right)-T_{y y_{i, j-1 / 2}}\left(\frac{h_{i, j}^{k+1}-h_{i, j}^{k+1}}{\Delta y}\right)}{\Delta y}$

Substitution of these equations into the governing equation, Eq. 14, leads to the finite difference approximation of the governing equation as

$F_{i j} h_{i, j}^{k+1}=A_{i j} h_{i+1, j}^{k+1}+B_{i j} h_{i, j-1}^{k+1}+C_{i j} h_{i-1, j}^{k+1}+D_{i j} h_{i, j+1}^{k+1}+E_{i j} h_{i, j}^{k}$

$$
\begin{aligned}
A_{i j} & =T_{x x_{i+1 / 2, j}} / \Delta x^{2} \\
B_{i j} & =T_{y y_{i, j-1 / 2}} / \Delta y^{2} \\
C_{i j} & =T_{x x_{i-1 / 2, j}} / \Delta x^{2} \\
D_{i j} & =T_{y y_{i, j+1 / 2}} / \Delta y^{2} \\
E_{i j} & =S_{i, j} / \Delta t \\
F_{i j} & =A_{i j}+B_{i j}+C_{i j}+D_{i j}+E_{i j}
\end{aligned}
$$

The boundaries and initial conditions are given by

$$
\begin{aligned}
& \frac{\partial h(x, y, t)}{\partial \mathbf{n}}=0 \quad \text { for } x, y \in \Gamma \quad(\text { no }- \text { flow condition }) \\
& h(0, y, t)=h_{0} \\
& h(L, y, t)=h(t)
\end{aligned}
$$

where $\Gamma$ is the lateral boundary and $\mathbf{n}$ is the unit vector normal to $\Gamma$ pointing out forward from the boundary.

\section{Appendix B: solution algorithm}

The procedure for solving the model equations involves the following steps between two successive time steps $\mathrm{k}$ and $\mathrm{k}+1$.

First iteration, denoted by $(0)$, for the time step $(\mathrm{k}+1)$ :

- An initial estimation of the heads $h_{i, j}^{k+1(0)}$ is given for all the nodes 
- The residual $r_{i j}^{k+1(0)}$ is computed as follows:

$$
\begin{aligned}
r_{i j}^{k+1(0)}= & A_{i j} h_{i+1, j}^{k+1(0)}+B_{i j} h_{i, j-1}^{k+1(0)}+C_{i j} h_{i-1, j}^{k+1(0)} \\
& +D_{i j} h_{i, j+1}^{k+1(0)}+E_{i j} h_{i, j}^{k(0)}-F_{i j} h_{i, j}^{k+1(0)}
\end{aligned}
$$

- A matrix $P_{i j}^{k+1(0)}$ is introduced as

$$
P_{i j}^{k(0)}=r_{i j}^{k(0)}
$$

- Another matrix $Q_{i j}{ }^{k+1(0)}$ is introduced as

$$
\begin{aligned}
Q_{i j}^{k+1(0)}= & F_{i j} r_{i, j}^{k+1(0)}-A_{i j} r_{i+1, j}^{k+1(0)}-B_{i j} r_{i, j-1}^{k+1(0)} \\
& -C_{i j} r_{i-1, j}^{k+1(0)}-D_{i j} r_{i, j+1}^{k+1(0)}+E_{i j} r_{i, j}^{k(0)}
\end{aligned}
$$

- The residual is summed over all the nodes

$$
\left|r^{k+1(0)}\right|^{2}=\sum_{i} \sum_{j}\left(r_{i j}^{k+1(0)}\right)^{2}
$$

- The inner product of $P_{i j}{ }^{k+1(0)}$ and $Q_{i j}{ }^{k+1(0)}$ is computed to evaluate the parameter $\alpha^{k+1(0)}$,

$\alpha^{k+1(0)}=\frac{\left|r^{k+1(0)}\right|^{2}}{\left(P^{k+1(0)}, P^{k+1(0)}\right)}$

- The heads and the residuals are updated

$$
\begin{aligned}
& h_{i j}^{k+1(1)}=h_{i j}^{k+1(0)}+\alpha^{k+1(0)} P_{i j}{ }^{k+1(0)} \\
& r_{i j}^{k+1(1)}=r_{i j}^{k+1(0)}-\alpha^{k+1(0)} Q_{i j}{ }^{k+1(0)}
\end{aligned}
$$

- A parameter $\beta^{k+1(0)}$ is computed

$$
\beta^{k+1(0)}=\frac{\left|r^{k+1(1)}\right|^{2}}{\left|r^{k+1(0)}\right|^{2}}
$$

- For the next iterations $(m+1)$, the matrix $P$ and $Q$ are updated as follows

$$
\begin{aligned}
P_{i j}^{k+1(m+1)}= & r_{i j}^{k+1(m+1)}+\beta^{k+1(m)} P_{i j}^{k+1(m)} \\
Q_{i j}^{k+1(m+1)}= & {\left[F_{i j} r_{i, j}^{k+1(m+1)}-A_{i j} r_{i+1, j}^{k+1(m+1)}-B_{i j} r_{i, j-1}^{k+1(m+1)}-C_{i j} r_{i-1, j}^{k+1(m+1)}-D_{i j} r_{i, j+1}^{k+1(m+1)}+E_{i j} r_{i, j}^{k+1(m+1)}\right] } \\
& +\beta^{k+1(m)} Q_{i j}^{k+1(m)}
\end{aligned}
$$

- The iteration counter is incremented for a new and better estimation of the heads, until the sum of the residual is less than the convergence criterion $\varepsilon$, which has been chosen a priori.

$$
\left|r^{k+1(m)}\right|^{2}<\varepsilon
$$

\section{References}

Ababou R, McLaughlin D, Gelhar LW, Tompson AFB (1989) Numerical simulation of three-dimensional saturated flow. Transp Porous Media 4(6):549-565

Ackerer P, Kinzelbach W (1985) Modélisation du transport de contaminant par la méthode de marche au hasard: influence des variations du champ d'écoulement au cours du temps sur la dispersion [Contaminant transport modeling by the random walk method: influence of temporal variations in the flow field on the dispersion]. In: Proc. of the Symposium on the Stochastic Approach to Subsurface Flow, Montvillargene, France, June 1985

Adams EE, Gelhar LW (1992) Field study of dispersion in a heterogeneous aquifer: 2, spatial moments analysis. Water Resour Res 28(12):3293-3307
Bear J (1972) Dynamics of fluids in porous media, American. Elsevier, Amsterdam, 764 pp

Boggs JM, Young SC, Beard LM, Gelhar LW, Rehfeldt KR, Adams EE (1992) Field study of dispersion in a heterogeneous aquifer. 1. Overview and site description. Water Resour Res 28 (12):3281-3291

Cirpka O, Attinger S (2003) Effective dispersion in heterogeneous media under random transient flow conditions. Water Resour Res 39:1257

Dagan G (1982) Analysis of flow through heterogeneous random aquifers. 2. Unsteady flow in confined formations. Water Resour Res 18(5):1571-1585

Dagan G (1984) Solute transport in heterogeneous porous formations. J Fluid Mech 145:151-177

Dagan G, Bellin A, Rubin Y (1996) Lagrangian analysis of transport in heterogeneous formations under transient flow conditions. Water Resour Res 32(4):891-899

Dentz M, Carrera J (2005) Effective solute transport in temporally fluctuating flow through heterogeneous media. Water Resour Res 41:W08414. doi:10.1029/2004WR003571

Deutsch CV, Journel AG (1998) GSLIB: geostatistical software library and user's guide, 2nd edn. Oxford University Press, New York

Elfeki AMM (1996) Stochastic characterization of geological heterogeneity and its impact on groundwater contaminant transport, PhD. Thesis, Delft University of Technology. Balkema, Rotterdam, The Netherlands, $301 \mathrm{pp}$

Elfeki AMM (2000) Stochastic models for characterizing variability of hydro geological parameters. In: Abo Rayan M (ed) IWTC 
2000, The Fifth International Water Technology Conference. Mansoura University Press, Mansoura, Egypt, pp 171-192

Elfeki AMM (2003) Transient groundwater flow in heterogeneous geological formations. Mansoura Eng J 28(1):c58-c67

Elfeki AMM, Uffink GJM, Barends FBJ (1996) Solute transport in single and multiple scale heterogeneous formations: numerical experiments. In: Soares A, Hernandez J, Froidevaux R (eds) geoENV 96, First European Conference on Geostatistics for Environmental Applications. Lisbon, Portugal, Kluwer, Dordrecht, The Netherlands, pp 51-63

Elfeki AMM, Uffink GJM, Barends FBJ (1997) Groundwater contaminant transport: impact of heterogeneous characterisation: a new view on dispersion. Balkema, Rotterdam, The Netherlands

Elfeki AMM, Uffink GJM, Lebreton S (2007) Simulation of solute transport under oscillating groundwater flow in homogeneous aquifers. J Hydraul Res 45(2):254-260

Freyberg DL (1986) A natural gradient experiment on solute transport in a sand aquifer: 2, spatial moments and the advection and the dispersion of non-reactive tracers. Water Resour Res 22 (13):2013-2046

Gelhar LW (1993) Stochastic subsurface hydrology. Prentice Hall, $\mathrm{NJ}$

Goode DJ, Konikow LF (1990) Apparent dispersion in transient groundwater. Water Resour Res 26(10):2339-2351

Haldorsen HH, Damsleth E (1990) Stochastic modelling. J Petrol Technol 42:404-412

Holly FM, Usseglio-Polatera J-M (1984) Dispersion simulation in two-dimensional tidal flow. J Hydraul Eng 110(7):905-926

Kinzelbach W (1986) Groundwater modelling. Elsevier, Amsterdam, 333 pp

List EJ, Gartrell G, Winant CD (1990) Diffusion and dispersion in coastal waters. J Hydraul Eng 116(10):1158-1179

Mantoglou A, Wilson JL (1982) The turning bands method for simulating of random fields using line generation by a spectral method. Water Resour Res 18(5):1379-1394
Rehfledt KR, Gelhar LW (1992) Stochastic analysis of dispersion in unsteady flow in heterogeneous aquifers. Water Resour Res 28 (8):2085-2099

Shinozuka M, Jan CM (1972) Digital simulation of random processes and its applications. J Sound Vib 25(1):111-128

Smith L (1981) Spatial variability of flow parameters in a stratified sand. Math Geol 13(1):1-12

Strikwerda JC (1989) Finite difference schemes and partial differential equations. Wadsworth and Brooks, Pacific Grove, CA

Sudicky EA (1986) A natural gradient experiment on solute transport in a sand aquifer: 2 , spatial moments and the advection and the dispersion of non-reactive tracers. Water Resour Res 22 (13):2069-2082

Tompson AFB, Gelhar LW (1990) Numerical simulation of solute transport in three-dimensional, randomly heterogeneous porous media. Water Resour Res 26(10):2541-2562

Uffink GJM (1990) Analysis of dispersion by the random walk method. PhD Thesis, Delft University of Technology, Delft, The Netherlands

Uffink GJM, Elfeki AMM, Lebreton S (2005) Simulation of dispersion in a heterogeneous aquifer: discussion of steady versus unsteady groundwater flow and uncertainty analysis. In: Vrijling JK, Rurijgh E, Stalenberg B, Van Gelder PHAIM, Verlaan M, Zijderveld A (eds) Proc. of the 9th International Symposium on Stochastic Hydraulics. Nijmegen, The Netherlands, 23-24 May 2005, papers on CD-ROM

Wang H, Anderson M (1982) Introduction to groundwater modeling. Freeman, San Francisco, $237 \mathrm{pp}$

Wang J, Tsay T-K (2001) Tidal effects on groundwater motions. Transp Porous Media 43:159-178

Wood BD, Kavvas ML (1999) Stochastic solute transport under unsteady flow conditions: comparison of theory, Monte Carlo simulations and field data. Water Resour Res 35(7):2069-2084

Yim CS, Mohsen MFN (1992) Simulation of tidal effects on contaminant transport in porous media. Ground Water 30(1):78-86 